\title{
Molecular identification and antifungal susceptibility testing of fungal agents isolated from patients with idiopathic pulmonary fibrosis(IPF) in Tehran, Iran
}

Maryam Roudbary*1, Hossein keyvani ${ }^{2}$, Maryam Esghaei ${ }^{2}$, Shahla Roudbarmohammadi ${ }^{3}$, SeyedAli Javad Mousavi ${ }^{4}$,Mahtab Ashrafi Khozani ${ }^{1}$, fatemeh Nikoomanesh ${ }^{3}$

1.Department of Medical Mycology and Parasitology,School of Medicine,Iran University of Medical Science, Tehran,Iran

2. Department of virology ,School of Medicine,Iran University of Medical Science,Tehran, Iran

3. Department of Mycology,Faculty of Medical Sciences,Tarbiat Modares University,Tehran,Iran

4. Department of Internal Medicine, School of Medicine, Iran University of Medical Sciences, Tehran, Iran

\begin{abstract}
Objectives
Idiopathic pulmonary fibrosis (IPF) is a chronic and progressive lung disease. Fungal colonization in patients with lung tissue damage cause of persistent infection. Fungal infections had been studied as causitive agents in cases with cystic fibrosis(CF) previously.It is expected there is a association between fungal agents and etiology of IPF. This study aimed to investigate the isolation and molecular indentification of fungal agents in IPF patients for the first time in Iran .
\end{abstract}

\section{Methods}

Forty nasopharyngeal (NP) swabs or bronchoalveolar lavage (BAL) samples were obtained from patients that were diagnosed by sophisticated practitioner from April 2015 to March 2016. Direct examination by hydroxide potassium $(\mathrm{KOH})$ carried out for the presence of fungal elements. The samples cultured on Sabauroud dextrose agar (SDA) medium.Conventional methodes, Polymerase Chain Reaction(PCR) and Sequencing were used for identification of fungal species. Antifungal susceptibility testing for yeast isolates which was conducted as standardized by Clinical Laboratory Standards Institute (CLSI M27- S3 and S4).

\section{Results:}

Of 40 IPF patients ,22(55\%) were female and 18(45\%) were male.7(17.5\%) of IPF patients were positive for fungal agents as following; 4 (10\%)Candida albicans(C. albicans), 2(5\%) Candida glabrata (C. glabrata)and 1(2.5\%) Aspergillus fumigatus( $A$. fumigatus) were identified using conventional methods(culture), PCR and sequencing technique. It was found the significant correlation between C.albicans colonization in upper respiratory system tract and presence underlying disease in IPF patients $(P<0.05)$. Antifungal susceptibility testing showed, all $C$. albicans isolates were resistant to itraconazole and it is found $3(75 \%)$ C.albicans were resistance to amphoterecin B, Whereas $3(75 \%)$ and $1(25 \%) \quad$ C. albicans isolates were susceptible dose dependant and resistant to fluconazole respectively. Morever, C. glabrata isolates were resistant to fluconazole, itraconazole and amphotricin B .

\section{Conclusions:}

Taken together, Fungal agents were detected in $17.5 \%$ of iranian IPF patients. Rregarding the high resistance Candida isolates against antifungal drugs, isolation and identification of fungal agents, as well as antifungal susceptibility testing is urgent necessity in IPF patients.Also clinician should be notice the antifungal prophylaxis in susceptible IPF cases, Howere the causes of IPF require more investigation.

Keywords:IPF,Candida,Aspergillus,Antifungal susceptibility testing 